\title{
Reportes de suicidios en prensa de Barranquilla: un enfoque desde salud pública
}

\author{
Reports of suicides in barranquilla press: an approach from \\ public health
}

Relatórios de suicídios em barranquilla press: uma abordagem de saúde pública

Jorge Bilbao Ramírez' , Adalgisa Alcocer Olacireguis², Leanette Lallemand Martínez², Jan Frank Lío Carrillo ${ }^{3}$, Rusvelt Vargas Moranth" $₫$

Fecha correspondencia: Recibido: febrero 3 de 2016. Revisado: abril 22 de 2016. Aceptado: mayo 10 de 2016.

Forma de citar:

Bilbao J, Alcocer A, Lallemand L, Lío J, Vargas R. Reportes de suicidios en prensa de Barranquilla: un enfoque desde salud pública. Rev CES Salud Pública. 2016; 7 (2): 1-9.

Open access

(C) Copyright

Licencia creative commons

Ética de publicaciones

Revisión por pares Gestión por Open Journal System DOl: http://dx.doi.org/10.21615/ cessp.7.2.1

ISSN: 2145-9932

Sobre los autores:

1. Médico, Sociólogo, Candidato a Doctorado en Ciencias de la Educación; Director SIDI Universidad Metropolitana; Docente investigador Universidad Libre de Barranquilla.

2. Magíster en Epidemiología, Docente investigadora Universidad Metropolitana.

Comparte

\section{Resumen}

Objetivo: Describir las características de los reportes de suicidio en medios de comunicación impresos de la ciudad de Barranquilla, durante 20082011. Materiales y métodos: se realizó un estudio descriptivo, transversal, con toma de muestra universal, donde se describen las distribuciones de frecuencias de cada una de las características de los reportes de suicidios publicados en las notas periodísticas en los medios de comunicación impresos de la ciudad de Barranquilla. Resultados: Se observó que el mayor índice de suicidio esta dado en el sexo masculino; se suicidan más personas dentro del rango de edad de 20 a 29 años (población juvenil), el medio de suicidio más utilizado fue el ahorcamiento. El horario que predominó fue en horas de la mañana. Un alto porcentaje no indicaba antecedentes de intentos previos, los cuerpos sin vida fueron hallados por familiares. Un alto porcentaje publicó fotos del fallecido. Las noticias estaban publicadas en primera página. Las líneas y los párrafos eran extensos; y las posibles causas eran atribuidas a trastornos depresivos. Los medios de comunicación no se rigen bajo las recomendaciones establecidas por la OMS. Conclusión: Las distribuciones de frecuencias de las características de los reportes de suicidios estudiados arrojaron porcentajes elevados, de importancia en salud pública.

Palabras clave: Suicidio, prensa, notificación.

\section{Abstract}

Aim: To describe the characteristics of suicide reports in press from the city of Barranquilla, during 2008-2011. Materials and methods: A descriptive, cross-sectional study, with universal sample, where there were described frequency distributions of the characteristics of reported suicides in news stories published in press. Results: It was observed that the highest rate of suicide is given in males; more people within the range of 20 to 29 years (young population) commit suicide, the most widely used means of suicide was hanging. The schedule was predominant in the morning. A high percentage did not indicate a history of previous attempts, the bodies were found by relatives. A high percentage published photos of the deceased. The news was published on the front page. Lines and paragraphs were ex- 
3. Médico cirujano Fundación Universitaria San Martín, sede Puerto Colombia.

4. Médico Cirujano. Magíster en Epidemiología. Docente investigador Universidad Metropolitana. tensive; and possible causes were attributed to depressive disorders. The media are not governed under the recommendations established by the WHO. Conclusion: The frequency distributions of the characteristics of reported suicides studied yielded high percentages of public health importance.

Keywords: Suicide, press, notice.

\section{Resumo}

Objetivo: descrever as características dos relatórios de suicídio na imprensa, na cidade de Barranquilla, durante 2008-2011. Materiais e métodos: Estudo descritivo, transversal foi realizado com amostra universal fazendo, onde as distribuições de cada uma das características de relatos de suicídios publicados em notícias na mídia impressa da cidade de frequência são descritos Barranquilla. Resultados: Foi observado que a maior taxa de suicídio é dada em machos; mais pessoas na faixa etária de 20 a 29 anos (jovens) cometer suicídio, o suicídio significa mais utilizado foi o enforcamento. 0 cronograma foi predominante na parte da manhã. Uma elevada percentagem não indicam uma história de tentativas anteriores, os corpos foram encontrados por parentes. Uma elevada percentagem publicou fotos dos falecidos. A notícia foi publicada na primeira página. Linhas e parágrafos foram extensa; e as possíveis causas foram atribuídas a transtornos depressivos. Os meios de comunicação não são regidos de acordo com as recomendações estabelecidas pela OMS.

Conclusão: As distribuições das características dos relatos de suicídios estudados frequência rendeu altas porcentagens de importância para a saúde pública.

Palavras-chave: Suicídio, Imprensa, notificação.

\section{Introducción}

La palabra "suicidio" proviene de las expresiones latinas suiv y occidere, que significan: el hecho de matarse a sí mismo. Es un fenómeno humano universal presente en todas las épocas, pero las distintas culturas han mantenido, actitudes variables hacia él, en función de principios filosóficos, religiosos e intelectuales (1). Diversas áreas del conocimiento han estudiado, debido a su crecimiento progresivo a través de los años, considerando su impacto social, familiar y personal.

El suicidio, una de las causas de muerte más frecuente, es el mayor problema de salud pública en países como los Estados Unidos. Según la Organización Mundial de la Salud, en la década anterior, el suicidio representaba un 1,8\% del total de las defunciones (2), con una tendencia hacia el alza que, de acuerdo con este organismo, originaría, el año 2020 alrededor de 1,53 millones de actos consumados, lo cual equivale a un promedio de muertes cada 20 segundos. A pesar de los esfuerzos en prevención e investigación, estos índices parecen no sufrir ninguna modificación, al punto que en países como Colombia la tasa se ha mantenido en 4 por cada 100.000 habitantes ( $)$.

El suicidio también es un fenómeno social que debe tratarse en conjunto y no sólo analizar los actos motivacionales que llevaron a cada individuo a cometerlo (ㄴ) . Desde este postulado y entendiendo que el factor precipitante no ocurre bajo una misma condición, sino que varía de persona a persona, es adecuado estudiar la percepción de los medios de comunicación, a través de las noticias de casos ocurridos en la ciudad. 
El suicidio es tal vez la forma más trágica de terminar con la propia vida. La mayoría de las personas que se plantean la idea del suicidio son ambivalentes. No están seguras de querer morir. Uno de los muchos factores que pueden llevar a una persona vulnerable al suicidio es la publicidad sobre este tema en los medios de comunicación. La forma como estos medios informan acerca de casos de suicidio puede influir en otros suicidios ( $\underline{5})$.

Desde la percepción de los actores sociales, el suicidio es una problemática multideterminada que involucra aspectos cognitivos, relacionales, educativos, económicos, políticos y culturales. Se destaca la necesidad de implementar políticas sociales integradas dirigidas a fomentar factores protectores como el Soporte social, la educación integral y la oferta de actividades para el uso del tiempo, e implementar programas que promuevan habilidades y competencias personales, junto con el diseño de estrategias conjuntas para reducir los factores de riesgo, cabe mencionar que la salud mental de la población es condición indispensable para el desarrollo social, por lo cual constituye una prioridad en salud pública (b).

Debido al aumento de los casos de suicidio los medios de comunicación optan por orientar su atención en estos hechos noticiosos, y no sería adecuado desconocer el derecho que tienen los comunicadores sociales o periodistas a difundir este tipo de información, como los ciudadanos de enterarse de estos temas, pero tampoco se debe dejar de lado la indagación, con una mirada crítica, acerca de si las noticias que se producen al respecto tienen la suficiente claridad conceptual sobre el manejo apropiado del tema, y si los periodistas son conscientes de la responsabilidad en el manejo del mismo. Más aún, si la redacción de este tipo de noticias no lesiona a los familiares o allegados, y si no incita a otras personas a cometer idéntica acción (b).

Los medios de comunicación juegan un papel significativo en la sociedad actual, al suministrar una amplia gama de información en variedad de formas. Ejercen una fuerte influencia sobre las actitudes, creencias y comportamientos de la comunidad y juegan un importante rol en la política, la economía y la práctica social. Debido a esa influencia, los medios de comunicación también pueden jugar un rol activo en la prevención del suicidio.

Sin embargo, existe gran preocupación entre la comunidad académica y la masa crítica sobre la forma como se presenta este tipo de noticias, el lenguaje utilizado y el manejo estilístico del hecho en la prensa escrita, máxime si se tiene en cuenta que los adolescentes y jóvenes son vulnerables a este fenómeno y, lo peor, se desconocen las sugerencias que hacen organismos como la Organización Mundial de la Salud (OMS) (7). Ante dicha percepción, es importante profundizar a cerca de la temática, observar cuál es el impacto de la noticia en la sociedad y enfatizar sobre el manejo adecuado de la información.

Existe evidencia suficiente para sugerir que algunas formas de cubrimiento periodístico y televisivo real del suicidio están asociadas con un exceso en suicidios estadísticamente significativo; el impacto parece ser mayor entre la gente joven. No obstante, los medios de comunicación no informan sobre la mayoría de los suicidios; cuando se toma la decisión de informar al público sobre un suicidio, usualmente involucra una persona, método o lugar en particular. Con frecuencia este tema es de interés periodístico y los medios de comunicación tienen derecho a informar sobre él. Desde hace años el suicidio se ha silenciado en la prensa por el conocido como "Efecto Werther" con este epónimo se conoce el aumento de los casos de 
suicidios que siguen a la aparición de historias sobre casos reales de suicidio en los periódicos y que se producen en áreas geográficamente próximas de distribución del medio. También se conoce como "efecto copycat", y es más probable que se produzca cuando el modelo es una persona célebre.

Es por eso que surge la necesidad de describir los suicidios en medios de comunicación impresos de la ciudad de Barranquilla, durante los años 2008-2011, debido a que no se han encontrado, hasta la fecha, estudios que enfaticen el manejo en la noticia en la prensa escrita de nuestra ciudad y el potencial impacto que esta genera en la sociedad.

\section{Materiales y métodos}

Se llevó a cabo un estudio descriptivo, transversal. Se revisaron 2922 ejemplares de los periódicos El heraldo (1461) y la Libertad (1461) de la ciudad de Barranquilla correspondientes a los años 2008-2011 en los cuales se encontraron 76 reportes en el periódico El heraldo y 69 en el periódico la Libertad. La muestra fue de tipo universal, ya que se describen todos los casos aparecidos en prensa escrita de los 2 medios de comunicación impresa de la ciudad de Barranquilla. La información se tomó de fuente secundaria, del archivo histórico de la hemeroteca de la Biblioteca Piloto del Caribe, y la recolección de la misma se llevó en un instrumento diseñado para tal fin.

La recolección de la información fue realizada por parte de los investigadores y de auxiliares de investigación previamente entrenados. Los datos fueron procesados en el programa Epiinfo versión 3.3.4, y el análisis se llevó a cabo principalmente mediante medidas de tipo descriptivo. Así mismo, en todo momento hubo respeto por la libertad y confidencialidad de los sujetos de estudio, según las Normas de Buenas Prácticas Clínicas en Investigación.

\section{Resultados}

La mayor proporción de casos reportados en El Heraldo se encontró durante el año 2008, con poco más de dos tercios de los 76, y en La Libertad el año con mayor reporte fue 2009 , con $44,9 \%$, y hubo meses en los que, mientras un diario reportaba casos, en el otro no aparecían, y llama la atención que, especialmente en el último trimestre de cada año, fue cuando se observó una mayor proporción de casos (tabla 1).

Tabla 1. Porcentajes de casos de suicidios reportados por los diarios La Libertad y El Heraldo, de la ciudad de Barranquilla, según mes de ocurrencia, durante el período 2008-2011.

\begin{tabular}{|c|c|c|c|c|c|c|c|c|}
\hline & \multicolumn{2}{|c|}{2008} & \multicolumn{2}{|c|}{2009} & \multicolumn{2}{|c|}{2010} & \multicolumn{2}{|c|}{2011} \\
\hline & $\begin{array}{c}\text { El } \\
\text { Heraldo } \\
(n=28)\end{array}$ & $\begin{array}{c}\text { La } \\
\text { Libertad } \\
(n=7)\end{array}$ & $\begin{array}{c}\text { El } \\
\text { Heraldo } \\
(n=23)\end{array}$ & $\begin{array}{c}\text { La } \\
\text { Libertad } \\
(n=31)\end{array}$ & $\begin{array}{c}\text { El } \\
\text { Heraldo } \\
(n=8)\end{array}$ & $\begin{array}{c}\text { La } \\
\text { Libertad } \\
(n=5)\end{array}$ & $\begin{array}{c}\text { El } \\
\text { Heraldo } \\
(n=17)\end{array}$ & $\begin{array}{c}\text { La } \\
\text { Libertad } \\
(n=26)\end{array}$ \\
\hline $\begin{array}{l}\text { Enero } \\
\text { Febrero } \\
\text { Marzo } \\
\text { Abril } \\
\text { Mayo } \\
\text { Junio } \\
\text { Julio } \\
\text { Agosto } \\
\text { Septiembre } \\
\text { Octubre } \\
\text { Noviembre } \\
\text { Diciembre }\end{array}$ & $\begin{array}{c}7,1 \\
0 \\
0 \\
0 \\
0 \\
3,6 \\
3,6 \\
17,9 \\
28,6 \\
25 \\
14,3 \\
0\end{array}$ & $\begin{array}{c}14 \\
0 \\
14 \\
0 \\
0 \\
0 \\
0 \\
0 \\
0 \\
0 \\
71 \\
0\end{array}$ & $\begin{array}{c}4,3 \\
4,3 \\
17,4 \\
17,4 \\
13 \\
13 \\
0 \\
0 \\
4,3 \\
4,3 \\
4,3 \\
17,4\end{array}$ & $\begin{array}{c}0 \\
6,5 \\
12,9 \\
19,4 \\
0 \\
16,1 \\
0 \\
0 \\
6,5 \\
16,1 \\
3,2 \\
19,4\end{array}$ & $\begin{array}{c}0 \\
0 \\
25 \\
0 \\
0 \\
25 \\
0 \\
12,5 \\
12,5 \\
0 \\
12,5 \\
12,5\end{array}$ & $\begin{array}{c}0 \\
40 \\
0 \\
0 \\
0 \\
0 \\
0 \\
0 \\
40 \\
0 \\
0 \\
20\end{array}$ & $\begin{array}{c}5,9 \\
11,8 \\
0 \\
0 \\
0 \\
5,9 \\
23,5 \\
5,9 \\
0 \\
0 \\
29,4 \\
17,6\end{array}$ & $\begin{array}{c}15,4 \\
3,8 \\
3,8 \\
11,5 \\
7,7 \\
3,8 \\
0 \\
19,2 \\
7,7 \\
3,8 \\
23,1 \\
0\end{array}$ \\
\hline
\end{tabular}

Fuente: Datos tomados por el grupo investigador. Archivos Hemeroteca Departamental. Biblioteca Piloto del Caribe 
Con respecto al área de residencia, $66 \%$ de los casos de El Heraldo correspondían a Barranquilla, así como $75,4 \%$ de los identificados en La Libertad, el resto procedían de fuera de la ciudad. Por género, la razón de masculinidad fue similar en ambos diarios: 6:1.

En cuanto a la distribución por edad, cabe resaltar que no todos los casos reportaron esta variable: $80,2 \%$ en El Heraldo y $89,8 \%$ en La Libertad. En ambos diarios la mayoría de casos con edad tenían entre 20 y 29 años: $25 \%$ en El Heraldo y 29\% en La Libertad, porcentaje seguido por el 15\% en el grupo de 40 a 49 años en el primero y 22,6\% para el grupo de 30 a 39 años en el segundo. Llama la atención que alrededor de 15\% de los casos en ambos diarios correspondían a adolescentes (10 a 19 años) (figura 1).

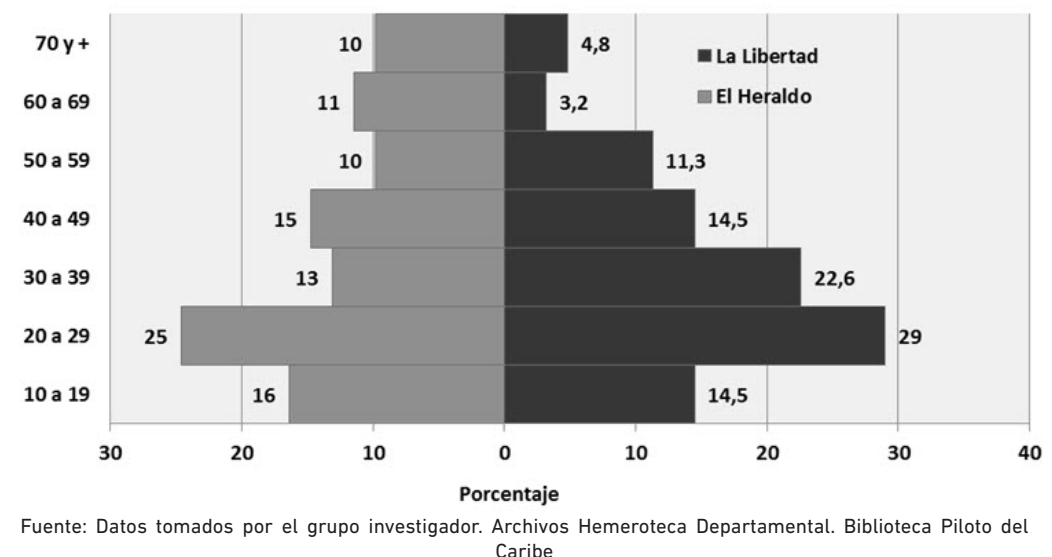

Figura1. Distribución de frecuencia de casos de suicidios según la edad reportados en ambos periódicos durante los años de estudio 2008-2011

Al analizar el horario, se encontró reporte del mismo en 42 casos en El Heraldo y 26 en La Libertad, de los cuales $47,6 \%$ y $42,3 \%$, respectivamente, se indicó que ocurrieron en la mañana, mientras que $21,4 \%$ y $26,9 \%$ se presentaron en la noche. El resto en la tarde y la madrugada.

Por otro lado, no se encontró información sobre el medio o forma de suicidarse, en 2 casos de El Heraldo y 3 casos de La Libertad. En el resto de reportes de prensa, se encontró que el principal medio fue el ahorcamiento: $71,6 \%$ y $74,2 \%$, seguido por arma de fuego $(12,2 \%$ y $12,1 \%)$ envenenamiento $(12,2 \%$ y $10,6 \%)$; el resto de casos correspondió, en El Heraldo, a 2 suicidios por caía de altura y uno por ahogamiento (inmersión), y en La Libertad a uno por caída de altura y otro por arma cortopunzante (tabla 2). De la misma manera, se encontró que, en las 76 notas periodísticas de El Heraldo el 7,9\% indicaba la existencia de intentos previos de suicidio, y en La Libertad este valor fue de 2,9\% (tabla 2). Así mismo, se encontró que 59 casos de $\mathrm{El}$ Heraldo y 58 de La Libertad mostraban información sobre la persona que encontró a la víctima, siendo principalmente familiares y amigos, con un porcentaje cercano al $90 \%$ en ambos diarios (tabla 2).

De otra parte, se encontraron descritos explícitamente nombres de familiares en $28,9 \%$ de los casos de El Heraldo y en 23,2\% de La Libertad, con mención de posibles causas en $65,8 \%$ y $69,6 \%$ de los reportes, respectivamente, correspondiendo principalmente a "depresión" para $26 \%$ y 33\%, seguido por "deudas" (20\% y 16,7\%). Llama la atención que en La Libertad en 4 casos hubo reseña respecto a la pérdida del año lectivo como posible causa del suicidio (tabla 2. 
Con respecto a los datos de la nota periodística, y su manera de presentación, se observó que mientras en El Heraldo 3,9\% de los casos estuvieron presentes en la primera página, en la Libertad esta cifra fue de 78,3\%; por otro lado, en 48,7\% de los casos de El Heraldo se mostró fotografía de la víctima, así como en 55,1\% de La Libertad, y al sumar categorías de la variable "número de párrafos", se observó que alrededor de $50 \%$ de los casos de La Libertad tenían más de cuatro párrafos, y en El Heraldo sólo 37,4\% (tabla 3).

Finalmente, se encontró que el total de los registros incumplen con al menos una las recomendaciones de la OMS para el reporte de los casos de suicidio, tanto para El Heraldo como para La Libertad.

Tabla 2. Porcentajes de casos de suicidios reportados por los diarios La Libertad y El Heraldo, de la ciudad de Barranquilla, durante el período 2008-2011, según características del caso descritas en la nota periodística

\begin{tabular}{|c|c|c|c|}
\hline & & $\begin{array}{c}\text { El Heraldo } \\
(n=74)\end{array}$ & $\begin{array}{l}\text { La Libertad } \\
\qquad(n=66)\end{array}$ \\
\hline \multirow{6}{*}{ Medio } & Ahorcamiento & 71,6 & 74,2 \\
\hline & Arma de Fuego & 12,2 & 12,1 \\
\hline & Envenenamiento & 12,2 & 10,6 \\
\hline & Caída de altura & 2,7 & 1,5 \\
\hline & Ahogamiento & 1,4 & 0,0 \\
\hline & Arma Cortopunzante & 0,0 & 1,5 \\
\hline \multirow{4}{*}{ Intentos previos } & & $(n=76)$ & $(n=69)$ \\
\hline & Sí & 7,9 & 2,9 \\
\hline & No indica & 92,1 & 97,1 \\
\hline & & $(n=59)$ & $(n=58)$ \\
\hline \multirow{4}{*}{$\begin{array}{l}\text { Persona que encontró } \\
\text { a la víctima }\end{array}$} & Familiar & 66,1 & 72,4 \\
\hline & Amigo & 20,3 & 20,7 \\
\hline & Autoridades & 10,2 & 5,2 \\
\hline & Vecino & 3,4 & 1,7 \\
\hline \multirow{4}{*}{ Nombres de familiares } & & $(n=76)$ & $(n=69)$ \\
\hline & Sí & 28,9 & 23,2 \\
\hline & No & 71,1 & 76,8 \\
\hline & & $(n=76)$ & $(n=69)$ \\
\hline \multirow{3}{*}{ Posibles causas } & Sí & 65,8 & 69,6 \\
\hline & No & 34,2 & 30,4 \\
\hline & & $(n=50)$ & $(n=48)$ \\
\hline \multirow{6}{*}{ Causas Descritas } & Depresión sin otra especificación & 26,0 & 33,3 \\
\hline & Deudas & 20,0 & 16,7 \\
\hline & Decepción amorosa/pasional & 18,0 & 10,4 \\
\hline & Problemas Conyugales & 10,0 & 12,5 \\
\hline & Pérdida año lectivo & 0,0 & 8,3 \\
\hline & Otras & 26,0 & 18,8 \\
\hline
\end{tabular}

Fuente: Datos tomados por el grupo investigador. Archivos Hemeroteca Departamental. Biblioteca Piloto del Caribe 
Tabla 3. Porcentajes de casos de suicidios reportados por los diarios La Libertad y El Heraldo, de la ciudad de Barranquilla, durante el período 2008-2011, según características de la noticia

\begin{tabular}{lccc}
\hline & & $\begin{array}{c}\text { El Heraldo } \\
(\mathbf{n}=74)\end{array}$ & $\begin{array}{c}\text { La Libertad } \\
(\mathbf{n}=\mathbf{6 6})\end{array}$ \\
\hline Primera página & Sí & 3,9 & 78,3 \\
& No & 96,1 & 21,7 \\
Foto del fallecido & Sí & 48,7 & 55,1 \\
& No & 51,3 & 44,9 \\
& $\mathbf{1}$ a $\mathbf{2}$ & 25,3 & 29 \\
Número & $\mathbf{3}$ a 4 & 37,3 & 21,7 \\
de párrafos & $\mathbf{5}$ a 7 & 24 & 18,8 \\
& $\mathbf{8}$ a 10 & 8 & 17,4 \\
\hline
\end{tabular}

Fuente: Datos tomados por el grupo investigador. Archivos Hemeroteca Departamental. Biblioteca Piloto del Caribe

\section{Discusión}

El suicidio es un problema importante de salud pública en todo el mundo. Según Hawton y van Heeringen es la décima causa de muerte de manera global (ㅇ). Sin embargo, esta información debe interpretarse de manera cautelosa, debido a la falta de concordancia en el análisis metodológico en las diferentes naciones. En todo caso, se estima que en las últimas cinco décadas las tasas han aumentado en un $60 \%$ con una importante proporción de jóvenes víctimas (9). De allí la importancia de su manejo, el cual debe ser integral y ético por parte de los medios de comunicación.

En el presente estudio se encontró una importante cifra de reportes de suicidios, la cual debe ser contrastada con datos oficiales provenientes de Medicina Legal y Ciencias Forenses, pero dan cuenta de un comportamiento un tanto disímil en el tiempo, ya que para los años 2008 y 2011 la mayoría de los casos se presentaron en el último trimestre, mientras que en 2010 hubo más casos registrados en el primer trimestre del año. Al respecto, en algunas partes del mundo se ha asociado el incremento en las tasas de suicidio con cambios estacionales, como el caso de Finlandia, en donde Hiltunen y cols., (10) encontraron que en hombres se presentaron más casos de suicidio en las temporadas más frías del año, o como en Estados Unidos, donde Bridges (11) reportó un incremento de casos relacionado con días de fiesta.

Con respecto a variables sociodemográficas como la edad y el sexo, en nuestro país las tendencias en las tasas de mortalidad por suicidios han sido mayores en hombres y han mostrado tasas específicas más elevadas en el grupo de 20 a 29 años, calificado como un segmento económicamente activo de la población (12), de manera similar a lo encontrado en el estudio mediante la revisión de los reportes de prensa.

Así mismo, elementos como las causas de lesión muestran un comportamiento semejante al de las estadísticas oficiales, ya que de acuerdo con Cendales y cols.,(12) al revisar cifras de Medicina Legal, los tres principales mecanismos de lesión en Colombia son: heridas por armas de fuego y explosivos, ahorcamiento y envenenamiento, con un incremento sostenido en el tiempo para los casos de ahorcamiento, lo cual, junto con los datos sociodemográficos, da cuenta de un grado importante de detalle en los artículos de prensa con respecto a la información de los casos de suicidio, tanto que en un porcentaje importante son incluidos datos confidenciales como los nombres de los familiares. 
El suicidio es de tal preocupación en la región, que entidades como la Secretaría de Salud del Departamento adelantan serios programas tendientes a frenar este flagelo; sin embargo, resaltan que los medios de información, especialmente los escritos, se hacen los de la vista ciega y en vez de contribuir a disminuir esta problemática sus publicaciones ocasionan, sin mesura y respeto, una figura de modelamiento que contribuye a que el suicidio se siga presentando (13).

Otros autores (14) han reportado que el suicidio tiene una variabilidad en las diferentes naciones donde se ha estudiado, pero es consistente en que los grupos de adolescentes y jóvenes representan un grupo de riesgo que hay que poner particular atención

Los medios de comunicación pueden jugar un papel proactivo en ayudar a prevenir el suicidio al publicar la siguiente información junto con noticias sobre el suicidio: Lista de servicios de salud mental y líneas telefónicas de ayuda disponibles con sus números telefónicos y direcciones actualizados, publicitar las señales de advertencia del comportamiento suicida, transmitir mensajes sobre la frecuente asociación entre la depresión y el comportamiento suicida, siendo la depresión una condición tratable, ofrecer un mensaje de solidaridad a los sobrevivientes en momentos de profundo dolor, proporcionando números de teléfono de los grupos de apoyo para sobrevivientes, si los hay. Esto aumenta la probabilidad de que profesionales en salud mental, amigos y familiares intervengan en las crisis suicidas (15).

\section{Conclusiones}

Las distribuciones de frecuencias de las características de los reportes de suicidios estudiados arrojaron porcentajes elevados, de importancia en salud pública.

\section{Bibliografía}

1. Bobes García J, González Seijo J, Sáiz Martínez PA. Prevención de las conductas suicidas y parasuicidas. Psiquiatría Médica. Editorial Masson; 1997 http://www.psiquiatria.com/sin-categoria/prevencion-de-las-conductas-suicidas-y-parasuicidas/\#

2. Organización Mundial de la Salud. El suicidio un problema de salud pública enorme. Boletín de Prensa Día mundial para la prevención del Suicidio. Ginebra, 2004. Tomado de: http://www.who.int/mediacentre/news/releases/2004/pr61/es/Fecha de acceso: Octubre 5 de 2015

3. Ceballos Ospino G. Características de las personas que consumaron suicidio en la ciudad de Santa Marta (Colombia) durante el año 2002: un informe de casos. Febrero de 2004. Asociación Colombiana para el avance de las ciencias del comportamiento. Tomado de: www.abacolombia.org. co. Fecha de acceso: Noviembre de 2012

4. Durkheim E. El Suicidio. Ediciones Akal. España: Madrid; 1995. https:// www.iberlibro.com/buscar-libro/titulo/el-suicidio/autor/durkheim/

5. Chmidtke A, Schaller S. What do we do about media effects on imitation of suicidal behaviour. In: De Leo D. Schmidtke A, Schaller S, eds. Suicide prevention: a holistic approach. Dordrecht, Kluwer Academic Publishers, 1998: 127-131. https://www. researchgate.net/publication/226042061 What do we Know about Media Effects on Imitation of Suicidal Behaviour 
6. Ojeda D, Villalobos F. Elementos para una Política Pública desde la Percepción del Suicidio en Nariño. Revista Colombiana de Psicología, Junio 2011; 20 (1): $57-$ 70 http://www.scielo.org.co/pdf/rcps/v20n1/v20n1a05.pdf

7. Rubiano H, Quintero T, Bonilla C. El suicidio: más que una noticia, un tema de responsabilidad social. Palabra clave, Diciembre 2007; 10(2): 93-111 http://www. redalyc.org/pdf/649/64910206.pdf

8. Hawton K, Van Heeringen, K. Suicide. Lancet, 2009; 373(1):1372-1381 http:// www.thelancet.com/journals/lancet/article/PIIS0140-6736(09)60372-X/ abstract

9. Hoven C, Mandell D, Bertolote J. Prevention of mental ill-health and suicide: public health perspectives. Eur Psychiatry, 2010; 25(1):252-256 https://www.ncbi.nlm.nih.gov/pubmed/20452753

10. Hiltunen L, Haukka J, Ruuhela R, Suominen K, Partonen T. Local daily temperatures, thermal seasons, and suicide rates in Finland from 1974 to 2010 . Environmental Health and Preventive Medicine. July 2014; 19(4): 286-294 https:// www.ncbi.nlm.nih.gov/pubmed/24794752

11. Bridges $S$. Rates of homicide and suicide on major national holidays. Psychological Reports, 2004; 94(1): 723-724. https://www.ncbi.nlm.nih.gov/ pubmed/15154207

12. Cendales R, Vanegas C, Fierro M, Córdoba R, Olarte A. Tendencias del suicidio en Colombia, 1985-2002. Revista Panamericana de Salud Pública. 2007; 22(4):231-238. http://www.scielosp.org/pdf/rpsp/v22n4/02.pdf

13. Gómez C, Rodríguez N, Bohórquez A, Diazgranados N, Ospina M, Fernández C. Factores asociados al intento de suicidio en la población colombiana. Revista de la Sociedad de Psiquiatría de Colombia, 2002; 31(4): 283-298 http://www.scielo. org.co/scielo.php?script=sci arttext\&pid=S0034-74502002000400002

14. Herrera A, Solórzano L, Aparicio B, Trinidad C. Suicidio en Centroamérica y República Dominicana, 1998-2008. Cuaderno de Psiquiatría Comunitaria, 2009; 9(2): 177-186 http://bddoc.csic.es:8080/detalles.html?id=675057\&bd=PSICOLO\&tabla $=$ docu

15. Wasserman D. Imitation and suicide: a reexamination of the Werther effect American sociological review, 1984; 49(1): 427-436. https://www.jstor.org/stable/2095285? seq=1\#page scan tab contents 